\title{
An SDN Framework for VANET
}

\author{
Shakeel Ahmed ${ }^{1}$ and Dr. Humaira Nishat ${ }^{2}$ \\ ${ }^{1}$ Sr. Asst. Professor, CVR College of Engineering/ECE Department, Hyderabad, India \\ Email: shakeel_be@yahoo.com \\ ${ }^{2}$ Professor, CVR College of Engineering/ECE Department, Hyderabad, India \\ Email: huma_nisha@yahoo.com
}

\begin{abstract}
Vehicular Ad hoc Networks (VANETs) have become the area of research and development since last few years. Communication today is taking place in moving vehicles due to advancement in technology. In VANETs, Vehicle to Vehicle (V2V) and Vehicle to Infrastructure (V2I) communication takes place. VANETs comprise of vehicles as nodes for the transfer of data in various situations. The routing protocols which were designed for many regular networks could not be applied to VANETs due to various unpredictable conditions. To communicate effectively through VANET, routing which has less delay and overhead is required. This paper gives a Software Defined Network (SDN) framework for efficient communication. Simulation results show that SDN framework improves the communication in terms of utilizing less delay.
\end{abstract}

Index Terms: data transmission, routing algorithms, SDN, VANETS.

\section{INTRODUCTION}

Vehicle Ad hoc Networks (VANETs) are a typical case of mobile ad hoc networks. They are formed between moving vehicles with vehicle to vehicle (V2V) communication or between vehicles and an infrastructure with vehicle to infrastructure (V2I) communication [1]. Vehicle industry has started using wireless communication technology so that vehicles can exchange data with other vehicles on the road [2]. VANET uses two basic communications V2V and V2I. To help this, Federal Communications Commission (FCC) dedicated $75 \mathrm{MHz}$ radio range in the $5.9 \mathrm{GHz}$ band [3]. These vehicles are dumped with many sensors and devices for $\mathrm{V} 2 \mathrm{~V}$ and V2I communication to prevent collisions, traffic monitoring etc. [4].

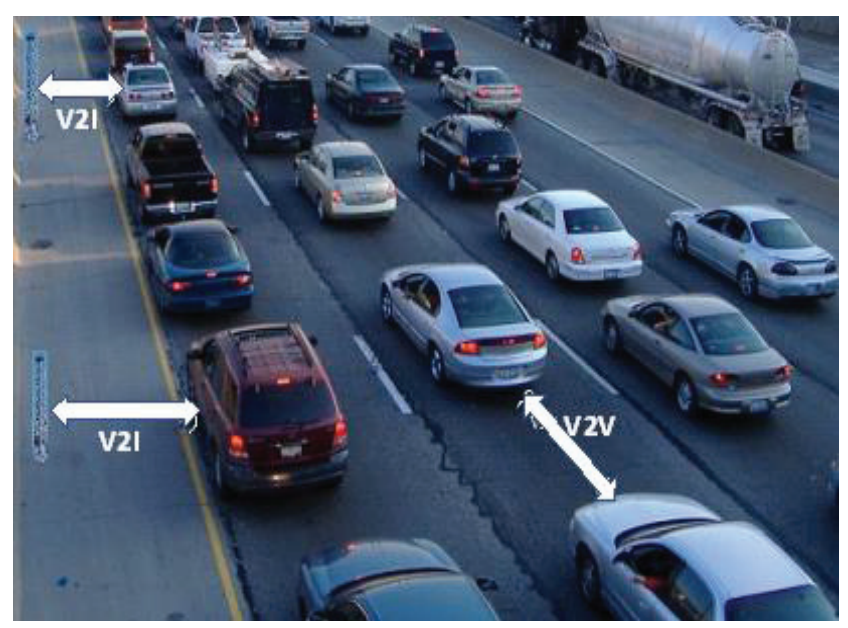

Figure 1. Vehicular Ad Hoc Networks
One of the typical VANET scenario is shown in figure 1. It shows $\mathrm{V} 2 \mathrm{~V}$ and V2I communication and also provides internet connectivity for the vehicles.

For VANET communication to happen between vehicles and roadside infrastructure units, vehicles are equipped with radio device or on-board unit that forms short-range wireless networks. Vehicles must also contain hardware that gives position details. Keeping all this in view communication device is installed in the vehicles which has variable speed of $30-180 \mathrm{~km} / \mathrm{h}$. Thus, varying speed gives unrealized challenges in designing system [5]. Not only the speed, but vehicles also mostly move on urban, rural and highways where they face rapid topological changes and changes in link characteristics which give a challenging task in finding the appropriate route at that movement.

.Issues in VANET include areas such as Quality Service (QoS), broadcasting, security attacks and routing. Now study is on the effects of transmission power on protocol performance and algorithms to control the power, and congestion control. The number of nodes also plays important role in calculating efficiency.

To increase the lifetime of network, scheduling schemes, power saving schemes can also be used [6]. VANET can be used for road safety, passenger easiness and now it is taken up in 5G technology to support traffic management.

This paper is organized as: section II gives an overall view of various routing protocols that can be used in VANET. In section III, a SDN framework is proposed for efficient communication among vehicles. Section IV gives description of simulation parameters. Section V discusses the results.

\section{Routing Protocols}

Vehicular Ad hoc Networks has unique characteristics of the network like dynamically changing topology, high mobility of vehicles and highly distributed network. The efficiency of routing protocols depends on various factors such as: internal - mobility of nodes and external - road topology and things that block the signal. This requires a highly changing approach to deal with such dynamic changing scenarios. Hence, selecting the best route and best strategies for forwarding is done by using various appropriate mobility and propagation models.

In vehicle to vehicle data transfer is one of the important challenges [7]. Routing in other ad hoc networks is different to the VANET routing because of extremely changing topologies. Thus, making routing focused area of research also routing play role in providing end to end reliable data transport with less routing overhead. The present routing protocols are modified to suite the VANETs. According to 
[8] routing in VANET can be classified as Topology based protocols and Position based protocols as shown in figure 2.

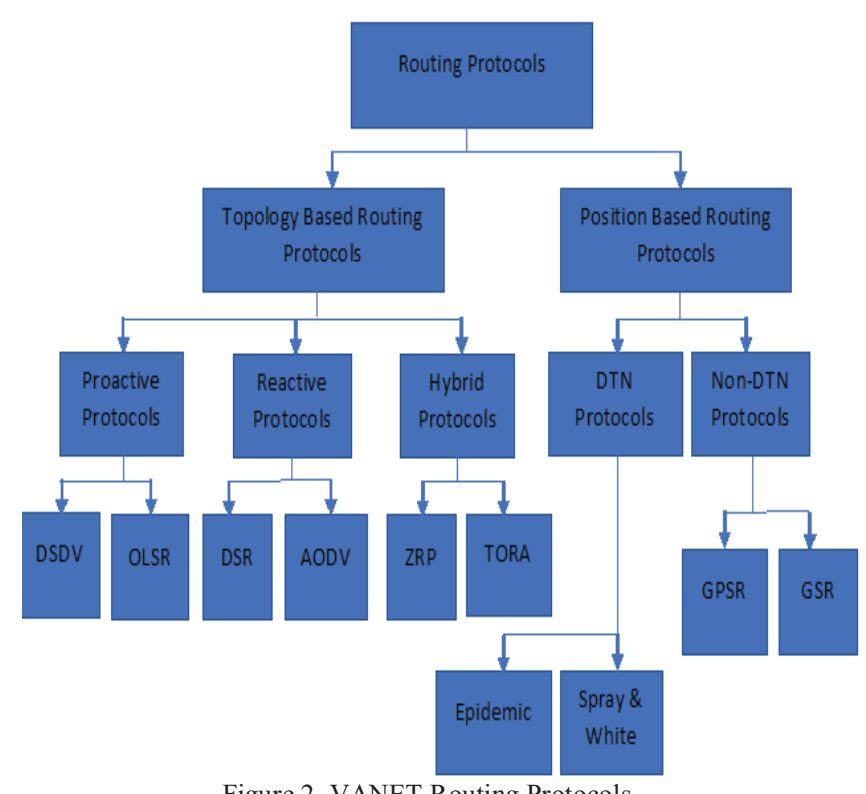

Figure 2. VANET Routing Protocols

\section{A. Topology Based Routing Protocols}

These protocols use link information to transmit data packets between nodes through the VANET. They are further divided into proactive, reactive and hybrid.

i) Proactive Routing Protocols

In the proactive routing protocols, all nodes maintain routing information periodically in the form of routing tables giving the complete network topological information. Advantage of proactive protocol is availability of routes since the route is stored in table. Disadvantage of this protocol is increased overhead and reduction in the available bandwidth. Because of this, proactive protocols cannot be used for high dynamic networks. Proactive routing protocols further classified as Destination-Sequenced Distance Vector (DSDV) and Optimized Link State Routing (OLSR)

DSDV is a distance vector routing protocol. In this each node uses Bellmon-Ford algorithm. The routing table is updated periodically with sequence number to avoid routing loops. The route gets highest and lowest sequence numbers. Highest sequence number route is preferred over the lowest, since in this also overhead is high. For a network with more frequently changing topology, this protocol is not suitable [9].

Optimized Link State Routing is based on state of a link. One of the features of DSDV is that it selects few nodes to act as relays which are called as Multipoint Relay (MPR). Because of MRP, flooding of data in the network is reduced thus reducing overhead [10].

\section{ii) Reactive Routing Protocols}

These are also known as on demand routing protocols which gets the route on demand. In this type when vehicle willing to transmit data, then only route is determined. Thus, reducing the unnecessary overhead. For establishing route it uses route discovery phase and route reply phase. In route discovery, the query packets are flooded into the network for the path search and in route reply phase, route is generated. As there are no regular updates of routing tables in the network, the bandwidth used is reduced. The disadvantage is it consumes more time to locate the routes as the source node should initiate route request process for data transmission. Thus, here the delay is increased. Examples are Dynamic Source Routing (DSR) and Ad hoc OnDemand Distance Vector (AODV).

DSR is a simple and efficient routing protocol which allows packets to be forwarded hop by hop. DSR also consists of route discovery and route maintenance phase. It is designed for mutti-hop networks. Here, the nodes keep caches to store intermediate nodes and destination information of each node. If any node fails, then it initiates route error packet [11].

AODV is the most widely used protocol in many networks. It supports both unicast and multicast routing. It has route discovery and route maintenance and maintains sequence numbers. AODV is combination of the concepts of both DSR \& DSDV. Route request is forwarded by in between vehicles which also generate a reverse route for destination. Processing delay is less due to this it can be employed for large networks [12].

\section{iii) Hybrid Routing Protocols}

The hybrid routing protocols combine proactive and reactive methods based on proactive protocols table maintenance and reactive protocols route discovery mechanisms. These routing protocols drawbacks are overcome and the good features of each is considered. Hybrid routing protocols are useful for a very large network size. The whole network is spit into smaller zones and proactive mechanism is implemented for intra zone communication and reactive mechanism is implemented for interzone communication. Examples are Zone Routing Protocol (ZRP) and Temporarily Ordered Routing Protocol (TORA)

ZRP is proposed to decrease the control overhead of proactive protocols and reduce the delay generated by the reactive protocols. In this, a zone is defined in terms of size and each node belongs to a particular zone within hop distance. ZRP is combination of two protocols, Intra-zone Routing Protocol (IARP) which is used inside zones and Inter-zone Routing Protocol (IERP) which is used between zones respectively. In this if the destination vehicle belongs to its own zone, then it delivers the packet directly. Otherwise, source vehicle broadcasts the Route Request to its peripheral nodes. The source node uses the path saved in the route reply packet to send data packets to the destination [13].

TORA is a hybrid protocol in which vehicles can be highly distributed. It uses two methods to update the information about the route: Query packet and Update packet. This routing algorithm aims to minimize the propagation of control packets and to minimize the responses to topological changes [14]. It also employs security authentication which is good. The disadvantage is the clock synchronization among vehicles. 


\section{B. Position Based Routing Protocols}

These protocols have the property of utilizing geographic location information to forward the data to next hop. The location information of vehicles could be monitored using Global Positioning System (GPS). The packet from the node is send without map information. The routing is carried out through the path selection, link estimation time, forwarding and recovery. These protocols are Delay Tolerant Network (DTN) and Non-delay tolerant network (Non-DTN) protocols.

\section{i) Delay Tolerant Network Routing Protocols}

The DTN architecture implements a store-and forward paradigm by overlaying a protocol. DTN routing protocols can endure huge delays and connection disruptions. DTN protocols are very much suitable substitute for traditional routing protocols in VANET. These protocols are further divided as Epidemic routing and Spray and Wait routing protocol.

Epidemic Routing protocol is implemented for message transmission in a frequently disconnecting network. The protocol basically uses flooding mechanism especially for wireless networks. It relies on exchanges of messages between nodes whenever they get in contact with each other to deliver the messages to their destinations.

Spray and Wait, a zero-knowledge routing protocol is introduced to reduce wasteful flooding of redundant messages. Spray and wait like epidemic routing, forwards copy of messages to other nodes randomly during connection in a network.

\section{ii) Non-delay tolerant network Routing Protocols}

Non-DTN protocols transmit a packet from source node to destination node as soon as possible. Examples are Greedy Perimeter Stateless Routing (GPSR) and Geographic Source Routing (GSR)

GPSR is a novel routing protocol for wireless network which uses the positions of vehicles and a packet's destination to take forwarding decisions. It uses only information about a vehicle's immediate neighbors in the network. When a packet does not find route, the algorithm recovers from the perimeter of the region. It keeps only the local topology state. When there are frequent topology changes, GPSR can utilize local information to find correct new routes as soon as possible. For small deployed networks, reliability cannot be promised.

GSR is based on the Dijkstra's routing algorithm. It will not flood the link state routing packets globally into the network. The main advantage is that route discovery and management is not required but it requires position determining services.

\section{SDN FRAMEWORK}

Software Defined Networking (SDN) is an emerging architecture that is dynamic, manageable and adaptable, making it ideal for the high-bandwidth, dynamic nature of today's applications [15]-[17]. Cloud Computing is a new field of research that aims to study mobile agents (people, vehicles, robots) as they interact and collaborate to sense the environment, process the data, propagate the results and more generally share resources.[18]-[20]. This architecture separates the network control and forwarding functions enabling the network control to become directly programmable.

Figure 3 shows the general architecture of SDN. SDN Applications are programs that explicitly, directly, and programmatically communicate their network requirements and desired network behavior to the SDN Controller. SDN is meant to address the fact that the static architecture of traditional networks is decentralized and complex while current networks require more flexibility and easy troubleshooting.

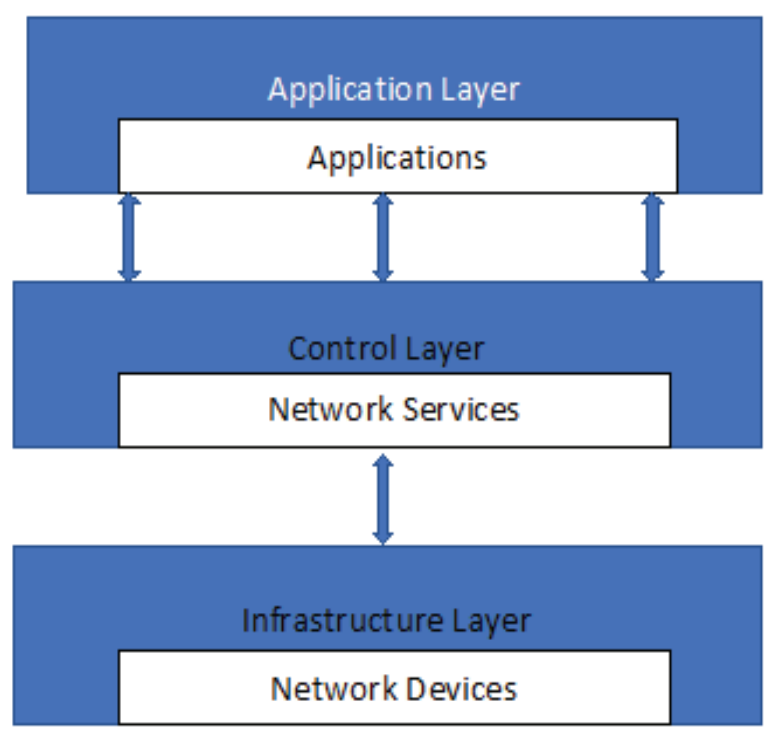

Figure 3. Simple SDN Architecture

SDN attempts to centralize network intelligence in one network component by disassociating the forwarding process of network packets into Infrastructure layer and the routing process into control Layer.

The SDN based framework for VANET is shown in figure 4 . Vehicles consist of vehicle to vehicle and vehicle to road side unit (RSU) communication devices. They also have Wifi devices and routing application. Wifi is used to transmit information to other vehicles and when it does not find route, it will send requirement for route to central controller. This in turn needs vehicle to send information through RSU to SDN controller. 


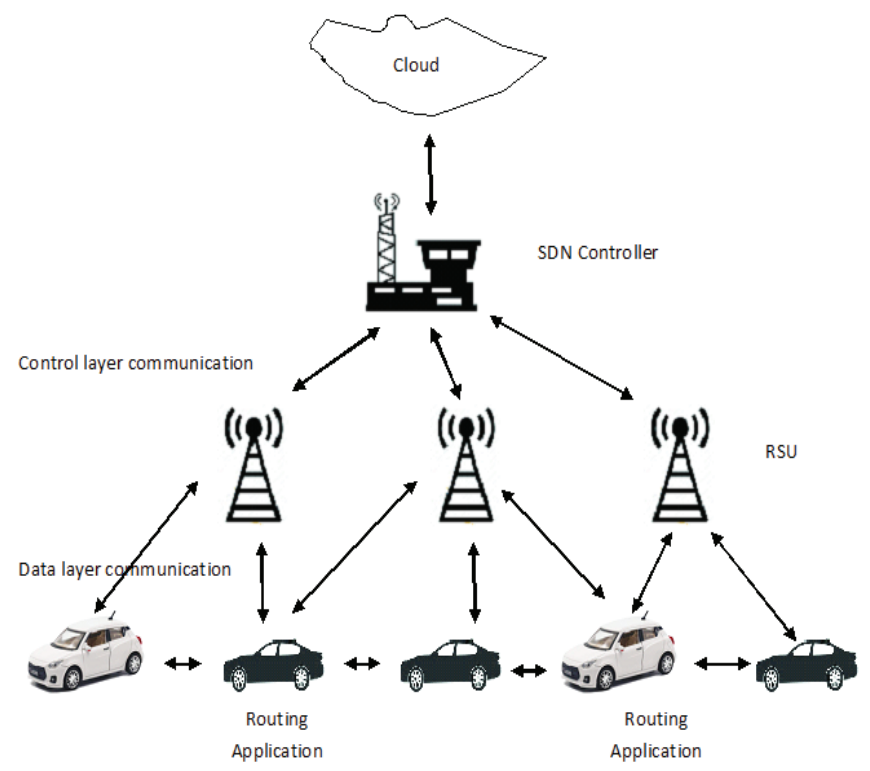

Figure 4. SDN Framework for VANET

SDN Controller has all knowledge of vehicle routes and RSUs. Thus, based on the requirement any one of the appropriate routing algorithm can be implemented in central controller with the help of Cloud.

\section{Simulation}

To evaluate the performance of SDN framework for VANET, some of the popular routing algorithms such as OLSR, AODV and TORA is considered. Simulation of Urban Mobility (SUMO) [21] is used generate the traces of vehicles in urban environment for light and heavy traffic test cases. The simulation parameters considered are as follows:

TABLE I.

SIMULATION PARAMETERS

\begin{tabular}{|l|l|}
\hline $\begin{array}{l}\text { Simulation } \\
\text { Parameters }\end{array}$ & Value \\
\hline Network area & $1000 \times 1000$ sqmts \\
\hline Packet size & 512 byte \\
\hline Traffic type & CBR \\
\hline MAC protocol & 802.11 \\
\hline Antenna & Omni directional \\
\hline Avg vehicle speed & $50 \mathrm{~m} / \mathrm{s}$ \\
\hline No. of Nodes & $1-100$ \\
\hline
\end{tabular}

\section{RESULTS}

The network performance is evaluated using light traffic condition i.e., 1 to 50 vehicles and heavy traffic condition 51-100 vehicles in the network area of $1000 \times 1000$ square meters.

The simulation of light traffic condition scenario where 18 nodes form a network and discover the route at the initial state is shown in figure 5.

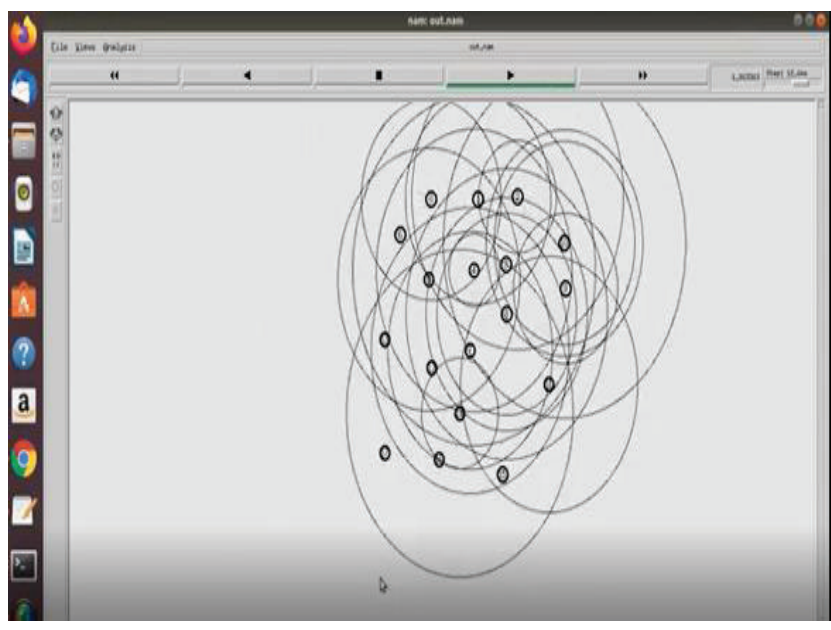

Figure 5. Route discovery in Light Traffic Scenario

Figure 6 shows light traffic condition where AODV routing protocol gives overall less packet delivery time for the various quantity of packets transmitted.

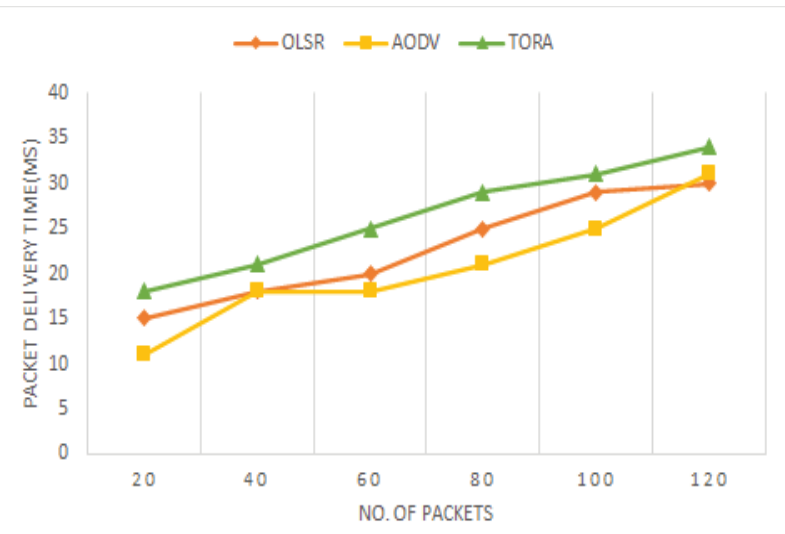

Figure 6. Light Traffic Scenario

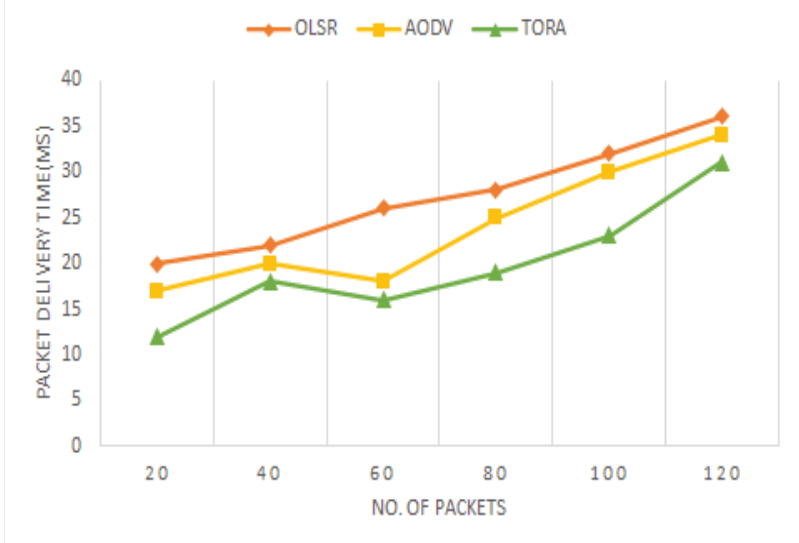

Figure 7. Heavy Traffic Scenario

In figure 7 , heavy traffic condition is in cooperated. All three protocols for average of 40 packets transmitted gives close packet delivery time whereas TORA routing protocol outperforms other protocols on an average. 


\section{CONCLUSIONS}

There is a demand now for efficient communication in VANET. This paper gives a SDN framework required for dynamic changing feature of VANET. Some of the standard protocols such as OLSR, AODV and TORA are compared in light and heavy traffic conditions in a limited area. It is concluded that in light traffic condition AODV on an average performs better and TORA protocol give better results that is less packet delivery time in high traffic conditions. In future, the protocols with security will be simulated and the various QoS metric will be evaluated.

\section{REFERENCES}

[1] Y. Toor, P. Muhlethaler, A. Laouiti, and A. D. L. Fortelle, "Vehicle ad hoc networks: applications and related technical issues," IEEE Communications Surveys Tutorials, vol. 10, no. 3, pp. 74-88, 2008.

[2] H. Hartenstein and L. P. Laberteaux, "A tutorial survey on vehicular ad hoc networks," IEEE Communications Magazine, vol. 46, no. 6, pp. 164-171, June 2008

[3] J. Choi, V. Marojevic, R. Nealy, J. H. Reed, and C. B. Dietrich, "DSRC and IEEE 802.11ac adjacent channel interference assessment for the5.9 GHz band,' in Proc. IEEE 89th Veh. Technol. Conf. (VTC-Spring), Kuala Lumpur, Malaysia, Apr. 2019, pp.1-5

[4] Wenshuang Liang, Zhuorong Li, Hongyang Zhang, Shenling Wang, and Rongfang Bie, "Vehicular Ad Hoc Networks: Architectures, Research Issues, Methodologies, Challenges, and Trends", International Journal of Distributed Sensor Networks, 2015.

[5] S. Zeadally, R. Hunt, Y. S. Chen, A. 1. and A. Hassan, "Vehicular ad hoc networks (VANETS): status, results, and challenges",Springer Science and Business Media, LLC 2010, pp. $217-241$.

[6] Nyoman Wira Prasetya; Tsang-Ling Sheu; Achmad Basuki; Muhammad Aziz Muslim, "A Pre-Emptive Traffic Model with Cluster-based TDMA for Vehicular Networks", IEEE International Conference on Software, Telecommunications and Computer Networks (SoftCOM), Sep. 2016, pp. 1-5.

[7] E.Royer and C.-K. Toh, "A Review of Current Routing Protocols for Ad Hoc Mobile Wireless Networks", IEEE Personal Communications Magazine, Vol.6, No. 2, April 1999.

[8] Ramandeep Kaur \& Chandan Sharma,"Review paper on performance analysis of AODV, DSDV, OLSR on the basis of packet delivery",IOSR Journal of Computer Engineering (IOSR-JCE), Volume 11, Issue 1 (May. -Jun. 2013), e-ISSN: 2278-0661, p- ISSN: 2278-8727

[9] Y. Fengjie, Y.Hui and Z.Ying," Research on DSDV routing protocol based on wireless mesh network", Chinese Control And Decision Conference, Shenyang, China, pp. 4292-4297, 2018.

[10] F. De Rango, M. Fotino and S. Marano, "EE-OLSR: Energy Efficient OLSR routing protocol for Mobile ad-hoc Networks," MILCOM 2008 - 2008 IEEE Military Communications Conference, San Diego, CA, USA, 2008, pp. 1-7, doi: 10.1109/MILCOM.2008.4753611.

[11] Mina Vajed Khiavi, Shahram Jamali \& Sajjad Jahanbakhsh Gudakahriz," Performance Comparison of AODV, DSDV, DSR and TORA Routing Protocols in MANETs "International Research Journal of Applied and Basic Sciences, Vol. 3(7), 1429-1436, 2012, ISSN 2251-838X

[12] Charles E. Perkins and Elizabeth M. Royer, "Ad hoc OnDemand Distance Vector Routing", Proceedings of the 2nd
IEEE Workshop on Mobile Computing Systems and Applications (WMCSA'99), pp. 90-100, February 1999.

[13] K. Yang and W. Huang, "Performance Research of an Improved Hybrid Routing Protocol," 2019 IEEE 19th International Conference on Communication Technology (ICCT), Xi'an, China, 2019, pp. 1242-1247

[14] Fang Yu, Yun Li, Fei Fang and Qianbin Chen, "A New TORA-based Energy Aware Routing Protocol in Mobile Ad Hoc Networks," 2007 3rd IEEE/IFIP International Conference in Central Asia on Internet, Tashkent, Uzbekistan, 2007, pp. 1-4, doi: 10.1109/CANET.2007.4401666.

[15] S. Bera, S. Misra, and A. V. Vasilakos, "Software-defined networking for internet of things: A survey," IEEE Internet of Things Journal, vol. 4, no. 6, pp. 1994-2008, 2017.

[16] D. Kreutz, F. M. V. Ramos, P. E. Verssimo, C. E. Rothenberg, S. Azodolmolky, and S. Uhlig, "Software-defined networking: A comprehensive survey," Proceedings of the IEEE, vol. 103, no. 1, pp. 14-76, Jan 2015.

[17] C. E. Palazzi, M. Roccetti, and S. Ferretti, "An intervehicular communication architecture for safety and entertainment," IEEE Transactions on Intelligent Transportation Systems, vol. 11, no. 1, pp. 90-99, March 2010.

[18] M. A. Salahuddin, A. Al-Fuqaha, and M. Guizani, "Softwarenetworking for RSU clouds in support of the Internet of vehicles," IEEE Internet Things J., vol. 2, no. 2, pp. 133_144, Apr. 2015.

[19] Park SC, Ryoo SY. An empirical investigation of end-users' switching toward cloud computing: A two factor theory perspective. Computers in Human Behavior 2013; 29(1):160 70.

[20] R. Gasmi and M. Aliouat, "Vehicular Ad Hoc NETworks versus Internet of Vehicles - A Comparative View," 2019 International Conference on Networking and Advanced Systems (ICNAS), Annaba, Algeria, pp. 1-6, 2019

[21] Simulation of Urban Mobility (SUMO). http://sumo-sim.org. 